\title{
Estudio Bibliométrico Sobre Intervenciones Psicológicas para la Esquizofrenia
}

\author{
Bibliometric Study on Psychological Interventions for Schizophrenia
}

\author{
Mónica Pachón-Basallo \\ Mónica Olarte-Afanador \\ Pontificia Universidad Javeriana Bogotá, Colombia \\ Wilson López-López \\ Pontificia Universidad Javeriana Bogotá, Colombia \\ Francisco José Santolaya-Ochando \\ Hospital Clínico Universitario de Valencia, España
}

(Rec: 18 de julio de 2013 / Acept: 9 de octubre de 2013)

\begin{abstract}
Resumen
La esquizofrenia es una enfermedad mental que, aun siendo abordada desde el siglo XVIII por Kraepelin y Bleuler, actualmente sigue causando desconcierto respecto a su curso y tratamiento. El objetivo de este trabajo era revelar con base en las investigaciones realizadas desde el año 2000 sobre psicoterapias para la esquizofrenia, la terapia que en la actualidad evidencia mayor eficacia para el funcionamiento integral de estos pacientes. Este estudio hizo uso de una metodología bibliométrica donde se analizaron un total de 71 artículos abstraídos de las siguientes bases de datos: Scopus, Pubmed, Medline, ScienceDirect, EbscoHost, Redalyc y Proquest. En los hallazgos encontrados, se evidenció que la terapia cognitivo comportamental fue utilizada en 30 de los 71 artículos. Terapias relacionadas como la cognitiva, y la cognitiva dirigida específicamente al manejo de habilidades sociales sumaron entre las dos otros 19 artículos. Estas terapias se destacaron por propiciar un tratamiento global para los pacientes.

Palabras clave: esquizofrenia, psicosis, enfoque cognitivo comportamental, bibliometría.
\end{abstract}

\begin{abstract}
Schizophrenia is a psychiatric illness that has been approached since the eighteenth century by Kraepelin and Bleuler, but continues to cause confusion with respect to its course and treatment. The objective of this study was to reveal, through research realized since 2000 on psychotherapy for schizophrenia, the therapy that with current evidence improves the efficacy for the integral function of these patients. This investigation was based on a bibliometric methodology that analyzed a total of 71 items abstracted from the following databases: Scopus, PubMed, Medline, ScienceDirect, EbscoHost, Redalyc and Proquest. The outcomes of the study demonstrated that cognitive behavioral therapy was used in 30 of the 71 articles analyzed. Related therapies, such as cognitive and cognitive specifically aimed at the management of social skills, added another 19 of these articles. These therapies were recognized for promoting an essential treatment for the patient. Key words: schizophrenia, psychosis, cognitive behavioral approach, bibliometrics.
\end{abstract}

\section{Introducción}

Tal vez no haya un reto más grande para un psicólogo que enfrentarse a un sujeto cuya realidad está encaminada bajo un mundo incomprensible para el resto de la sociedad. Desde el siglo XIX surgió el interés por la observación empírica de la naturaleza, esta abandonaba las teorías acerca de lo sobrenatural, convirtiendo a las ciencias Médicas en disciplinas científicas dado que los profesionales dedicados a las enfermedades mentales comenzaron a buscar un sistema diagnóstico coherente que fuera lógico y que les permitiera identificar, aislar y predecir las enfermedades y 
sus evoluciones. En 1809 el francés Philippe Pinel habló de demencia en los jóvenes, para que luego en 1814 su discípulo Jean Etienne Esquirol describiera cuadros emparentados llamándolos Demencia Juvenil, refiriéndose a la abolición del pensamiento lógico en los jóvenes. Sin embargo, fue Benedict Augustin Morel el que por primera vez presenta el nombre de Demencia Precoz a dichos cuadros (FusarPoli y Politi, 2008).

La concepción de esquizofrenia surgió desde Kraepelin en 1896, quien desde la psiquiatría habló por primera vez de la enfermedad con el término demencia precoz, delimitando varios trastornos dentro de la misma. Este, desarrolló diferentes textos sobre el tema e identificó un grupo de jóvenes que enfermaban y presentaban alucinaciones, delirios, movimientos anormales, empobrecimiento emocional, aislamiento social, entre otras, volviéndose completamente incapaces de atender y afectando significativamente al sujeto.

No obstante, Eugen Bleuler en 1908 en una conferencia de la Asociación Alemana de Psiquiatría en Berlín, acuñó el término de esquizofrenia, realizando una crítica a la connotación de Kraepelin, puesto que lo que él había observado en sus pacientes no se relacionaba estrictamente con una demencia, ni con personas jóvenes. Bleuler rescató y yuxtapuso dos conceptos griegos; schizen "dividir" y phren "alma, espíritu, de la mente" (Fusar-Poli y Politi, 2008).

Dentro de los factores de riesgo para la adquisición de la enfermedad, Gejman, y Sanders (2012), mencionan que se pueden presentar desde el período de desarrollo cerebral hasta otras situaciones que se presenten durante la adolescencia, la mayoría de estos factores actúan durante el embarazo o el parto. También se encuentran otros factores como el traumatismo craneano (Molloy et al., 2011), la epilepsia, las convulsiones febriles en niños (Vestergaard et al., 2005) o deformaciones serias (Dalman et al., 1999). Sin embargo, a la hora de enfrentarse al factor de riesgo individual se debe enfatizar en la historia familiar: esta, entendida como los antecedentes de la enfermedad en algún miembro familiar (Mortensen et al., 1999).

Asímismo, como menciona Pérez (2012), se suman múltiples factores; como pautas de crianza, patrones comunicacionales y experiencias traumáticas. Frente a estas últimas, se hace énfasis en los traumas infantiles, las investigaciones arrojan una clara correlación entre éstas y la aparición de la enfermedad (Read et al., 2005).

Actualmente, se encuentra una escala clínica que permite identificar personas con alto riesgo de desarrollar una enfermedad psicótica. Como mencionan Díaz y colaboradores (2010), esta fue creada por el grupo PACE (Personal Assessment and Crisis Evaluation), y trabaja con jóvenes entre los 14 y 29 años de edad. En ella se clasifica un grupo de síntomas psicóticos atenuados, donde el sujeto ha experimentado síntomas durante el último año.

Sin embargo, es difícil trabajar con sujetos que se encuentran en riesgo de presentar esta enfermedad, Morrison y colaboradores (2007) trabajaron en el EDIE (Early Detection and Intervention Evaluation Trial) y demostraron que aunque no se pueda controlar con precisión quiénes desarrollarán o no la enfermedad, al realizar un seguimiento durante tres años a un grupo control en terapia cognitiva, se permitió evidenciar que aquellos que presentaron la enfermedad, tuvieron un mejor control frente a sus pensamientos, miedos al rechazo y una reducción significativa frente a la prescripción de antipsicóticos.

Como evidencia lo anteriormente escrito, la psicoterapia es fundamental para el amoldamiento de estos nuevos pacientes a sus realidades y a la sociedad, por eso se hace necesario tener cada vez más certeza frente las terapias que hoy en día están demostrando mayor eficacia.

\section{Método}

\section{Tipo de estudio}

El estudio bibliométrico es una herramienta metodológica que parte de la necesidad de cuantificar ciertos aspectos de la ciencia (López,1996), parte de la cienciometría que aplica métodos matemáticos y estadísticos a toda la literatura de carácter científico, con el objetivo de estudiar y analizar la actividad científica, logrando cuantificar aspectos sociales de la ciencia (López, 1996) que, según Montero y León citados en Navarrete, Fernández, López, Quevedo y Buela (2009), facilitan un estudio descriptivo mediante un análisis de documentos.

Este tipo de metodología proporciona bloques de construcción empírica de la teórica, es decir pretende estudiar una serie de indicadores tomados de una población de documentos, logrando que sus resultados señalen lo que necesita ser explicado, permitiendo que las conclusiones tengan mayor relevancia científica (Schinka y Velicer, 2003).

\section{Muestra}

En un primer momento de la investigación se revisaron un total de 131 artículos, de los cuales 60 fueron excluidos por año de publicación, ausencia de datos específicos (nombre de revista de publicación), idiomas distintos al inglés o al castellano y falta de consistencia metodológica en los 


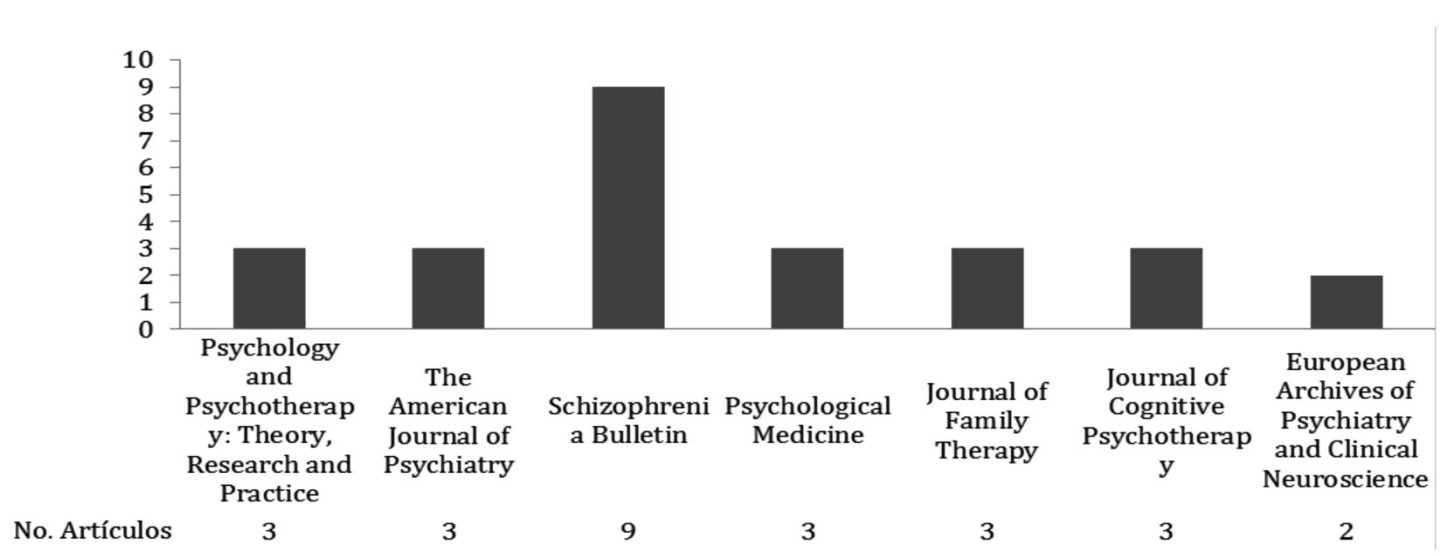

Figura 1. Revistas con más de una publicación analizada.

estudios (no aclarar objetivos, metodología, resultados o conclusiones del estudio).

\section{Procedimiento}

Se realizó una recolección de artículos que trabajan con psicoterapias dirigidas a pacientes con esquizofrenia, los cuales fueron abstraídos de bases de datos seleccionadas de acuerdo a su cobertura temática y regional. Se tuvieron en cuenta Scopus (siendo esta la base de datos que mayor impacto científico tiene en la actualidad dado a su cobertura temática) (García, Guerrero y Moya, 2012), Pubmed, Medline, ScienceDirect, EbscoHost, Proquest y Redalyc, (siendo esta última, una base de datos que se caracteriza por la cobertura significativa de revistas científicas iberoamericanas).

Se excluyeron de la muestra aquellos artículos que hacían referencia a esquizofrenia de tipo catatónico, puesto que su tratamiento psicológico difiere de los otros en relación con la relevancia del manejo de síntomas positivos y negativos.

Luego de haber seleccionado los artículos que cumplían con las características anteriormente mencionadas, se eligieron los siguientes datos de cada artículo: datos generales (año, país, autores, título, revista y datos específicos), tipo de terapia (cognitivo comportamental, psicodinámica, en habilidades sociales, psicoterapia, terapias múltiples, psicosocial, y otras) medicamentos (medicado, no medicado, qué medicamentos), tipo de investigación (cuasi-experimental, teórico, otros), enfoque metodológico (cualitativo, cuantitativo, mixto), tipo de muestreo, participantes, (cantidad, raza, sexo, edad, condición (interno o ambulatorio) y procedimiento (desarrollo, estadísticos y resultados).

Dado a los objetivos de esta investigación se utilizaron tres tablas de recolección de datos que permitieron vislumbrar de una manera clara y concisa aspectos relevantes de cada uno de los estudios encontrados además de los datos elegidos y explicados anteriormente.

\section{Resultados}

\section{Artículos por año}

Teniendo en cuenta los criterios de inclusión mencionados anteriormente, se encontraron en total 71 artículos de diferentes revistas incluidas en las bases de datos nombradas en el procedimiento. Estos, fueron publicados en los últimos trece años. Se evidenció, que especialmente en los años 2006 y 2007 se publicaron más artículos en comparación con los otros años.

\section{Revistas}

Así bien, las revistas en las que se analizó más de un artículo se representan en la figura 1, siendo Schizophrenia Bulletin con un total de nueve publicaciones, la revista de la que se estudiaron más artículos para la presente investigación.

Estos artículos corresponden a quince paises, entre los que se destacan por mayor cantidad de publicaciones Estados Unidos (29), Reino Unido (14) y España (6). En total, el $49 \%$ de los estudios analizados corresponden a América, el 47\% a Europa, el 3\% a Asía y el 1\% a Oceanía (Figura 2).

\section{Enfoque de investigación}

Profundizando en el contenido de los artículos analizados, se pudo evidenciar que los enfoques de investigación estuvieron divididos de la siguiente manera: Mixto $27 \%$ con 


\section{Ubicación de publicaciones}

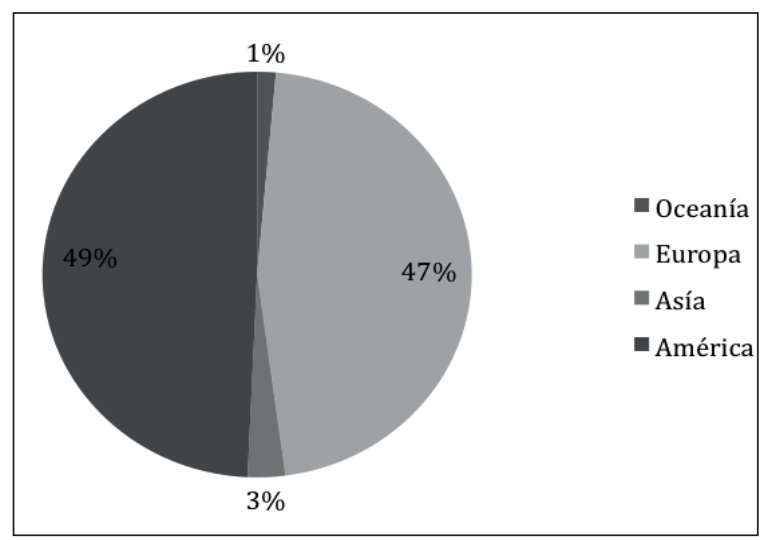

Figura 2. Porcentaje de publicaciones según continentes.

un total de 19 artículos, cuantitativo $48 \%$ con un total de 34 artículos y cualitativo $25 \%$ con un total de 18 artículos.

\section{Tipo de terapias psicológicas}

Para el análisis dirigido al tipo de terapia psicológica utilizada en los estudios, se tuvo en cuenta los marcos teóricos referenciados y las especificaciones de los autores respecto al proceso terapéutico llevado a cabo en cada caso específico. Es por eso que no se agrupó a criterio de los investigadores terapias que se relacionan a un mismo paradigma epistemológico, sino que se realizó el análisis según lo descrito en cada investigación.

En los hallazgos encontrados en esta investigación, se evidenció que la terapia cognitivo comportamental fue utilizada en 30 de los 71 artículos analizados; terapias relacionadas como la cognitiva, y la cognitiva dirigida específicamente al manejo de habilidades sociales sumaron entre las dos otros 19 artículos. Estas terapias en rasgos generales trabajaron en el funcionamiento global de los pacientes, comunicación asertiva, solución de problemas, discriminación de estímulos para pacientes con síntomas positivos, neutralización de estado de ánimo para pacientes con síntomas negativos, entrenamiento en habilidades sociales, rehabilitación cognitiva, estrategias de afrontamiento, adherencia al tratamiento, prevención de recaída hospitalaria, creencias frente a la enfermedad, regulación y autocontrol emocional, relación terapéutica, psicoeducación, calidad de vida, autocuidado, fortalecimiento de vínculos afectivos y en métodos de exposición. Algunos de estos estudios también trabajaron con familia y grupos de apoyo pero se analizaron desde el enfoque comportamental, puesto que los autores exponen que es el eje del tratamiento (Belanger, 2012; Lincoln, et al., 2012; Eack, 2012; Hasson-Ohayon 2012; Klingberg, et al., 2011; Fowler 2011; Wolwer y Frommann, 2011; Valencia et al., 2010; Virgil y Gregory, 2010; Jones, Cormac, Silveira da Mota Neto y Campbell, 2010; Onwumere et al., 2009; Garety et al., 2009; Christodoulides et al., 2008; Roder, Mueller y Schmidt, 2008; Kurtz y Mueser, 2008; Mak, Li y Lee, 2007; Penn, Roberts, Combs y Sterne, 2007; García-Bóveda 2007; Kingdon, Rathod, Hansen, Naeem y Wright, 2007; García, Ruiz, Roder y Soler, 2007; Everitt, Steel, Tarrier y Wykes, 2007; Glynnt, Cohen y Niv, 2007; Lysaker, Davis y Beattie, 2006; Naeem, Kingdon y Turkington, 2006; Bechdolf et al., 2006; Fadden, 2006; Bauml, Frobose, Kraemer, Rentrop y Pitschel-Walz, 2006; Cuevas, 2006; McCayand et al., 2006; Zimmermann, Favrod y Trieu, 2005; Cathera Pennb y Ottoa,

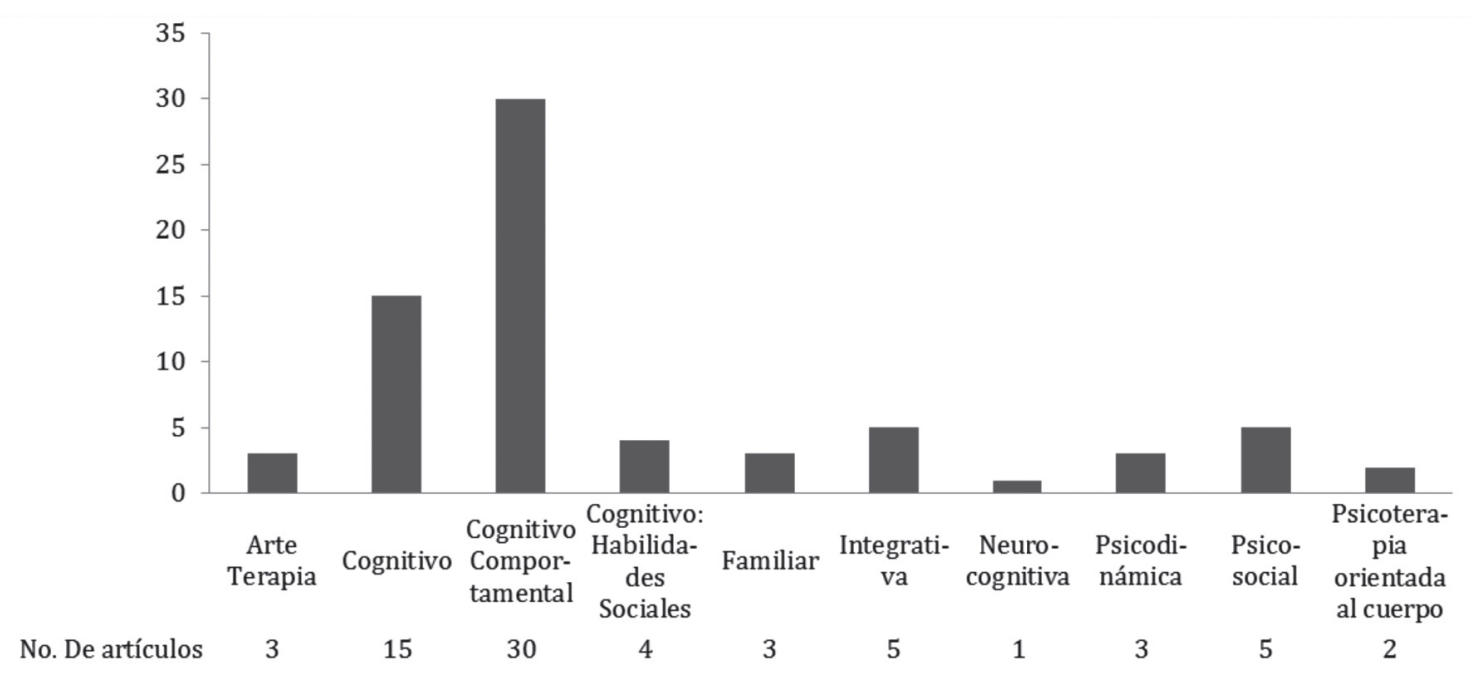

Figura 3. Tipo de terapias utilizadas en los artículos analizados. 
2005; Addington y Gleeson, 2005; Granholm et al., 2005; Weisman, 2005; Temple, 2004; Buckley, Pettit y Adams, 2004; Penn et al., 2004; Gumley et al., 2003; Twamley, Jeste y Bellack, 2003; Mohiuddin, Ahmed y Boisvert, 2003; Liberman, Glynn, Blair, Ross y Marder, 2002; Van der Qaag, Kern, Van den Bosch y Liberman, 2002; Pilling et al., 2002; Zygmunt, Olfson, Boyer y Mechanic, 2002; Glyn et al., 2002; Brenner, Pfammatter, 2000; Spaulding, Reed, Sullivan, Richardson y Weile, 2000).

Así bien, las terapias integrativas en su mayoría utilizaron herramientas de psicoterapias humanistas, comportamentales, dinámicas, constructivistas y psicodinámicas, (Schmidt-Kraepelin, Janssen y Gaebel, 2009; Garcia, 2008; Lysaker y Hermans, 2007; Lysaker y Daroyanni, 2006; Lysaker y Buck, 2006) éstas fueron utilizadas en 5 de los artículos analizados.

Frente a terapia familiar se analizaron tres artículos, estos enfocados en las relaciones de los pacientes con sus círculos de apoyo primarios, entendido el involucramiento de las familias en beneficios de adherencia al tratamiento por parte del paciente, también se trabajó en psicoeducación y en habilidades de comunicación efectivas. (Deane, Mercer, Talyarkhan, Lambert y Pickard, 2012; Fritze, Förthner, Schmitt y Thaler, 2007; Weisman, Duarte, Koneru y Wasserman, 2006).

Por otro lado, en terapia neurocognitiva, se analizó un artículo escrito por Vaskinn et al., (2008) en el que se estudió la relación frente a las funciones neuropsicológicas y la esquizofrenia, se trabajó en rehabilitación de funciones ejecutivas.

Frente a terapia psicosocial, se analizaron cinco artículos que se centraron en apoyo familiar, psicoeducación y alianza terapéutica (Smerud y Rosenfarb, 2011; Lysaker, Buck y LaRocco, 2007; Walkup, Wei, Sambamoorthi, Crystal y Yanos, 2006; Ran et al., 2003; Valencia, 2000).

Respecto a la psicoterapia orientada al cuerpo se analizaron dos artículos en los que se trabajó en grupo y se analizaron las implicaciones de ejercicios físicos en egopatología y síntomas negativos (Priebe et al., 2013; Röhricht, Papadopoulos, Suzuki y Priebe, 2009).

Frente al arteterapia se analizaron tres artículos, en estos se analizó la implementación de la música, el dibujo, y la expresión artística en general como fuente de contenidos simbólicos que permitieron que los pacientes encontraran nuevas formas de conexión y de interpretación frente a la enfermedad (La Rotta, 2012; Crawford et al., 2010; Reyes, 2007).

Finalmente, frente a terapias psicodinámicas se analizaron tres artículos, desde este enfoque se trabajó en la relación terapéutica, hipnosis, habilidades de comunicación, disminución de conductas problema y uso de medicamentos (Salamero y Manzano, 2005; Guseva, Iovlev y Shchelkove, 2002; Rogan, 2000).

\section{Diseño metodológico}

Respecto al diseño de investigación, el $60 \%$ de los artículos analizados es decir 43 de los 71 fueron cuasiexperimentales, estos estudios se caracterizaron por realizar un análisis mediante diferentes pruebas respecto al impacto de las diferentes intervenciones realizadas. El 27\% de los artículos analizados fueron descriptivos (19 artículos), los investigadores realizaron una descripción cualitativa del proceso y de los resultados obtenidos mediante los procesos psicoterapéuticos. Finalmente, el 13\% de los artículos analizados fueron teóricos ( 9 artículos). En estos, los autores realizaron diferentes análisis frente hallazgos teóricos y/o empíricos frente a la esquizofrenia.

\section{Tipo de muestreo}

El muestreo de los estudios analizados se dividieron en caso único (10\%) utilizado en siete de los artículos analizados, y por conveniencia (90\%), utilizado en 64 artículos. En la tabla 1 se especifica la cantidad de participantes totales y/o artículos utilizados en las investigaciones de índole teórica.

Tabla 1. Cantidad de participantes y estudios utilizados en las investigaciones consultadas

\begin{tabular}{|c|c|}
\hline $\begin{array}{l}\text { No. Estudios que especifican } \\
\text { número de participantes. }\end{array}$ & $\begin{array}{c}\mathrm{N} .^{\circ} \mathrm{De} \\
\text { participantes } \\
\text { total }\end{array}$ \\
\hline
\end{tabular}

No. Estudios teóricos que especifican número de artículos utilizados.

7

N. ${ }^{\circ}$ Artículos 3627

No. Estudios que no especifican cifras.

Total Estudios 71

Se pudo evidenciar que la mayoría de artículos definieron el número de participantes totales con el que trabajaron, 19 estudios no especificaron la cantidad de participantes. Frente a estudios teóricos analizados, siete especificaron la cantidad de estudios con los que se trabajó en sus investigaciones (tabla 1).

La edad de estos participantes se muestra en la tabla 2, los artículos analizados definían este criterio por edad 
exacta o por rangos. Se comprobó que la edad promedio de los participantes era de 38 años, y que en aquellos estudios en los que se estableció un rango de edad como criterio de inclusión, osciló entre los 18-60 años.

Tabla 2. Edad de participantes.

\begin{tabular}{lcc}
\hline Estudios que especifican edad & 23 & $\begin{array}{c}\text { Edad promedio } \\
38.14\end{array}$ \\
\hline Estudios que especifican rango & 18 & $\begin{array}{c}\text { Rango promedio } \\
18-60\end{array}$ \\
N.A & 30 & \\
Total Estudios & 71 & \\
\hline
\end{tabular}

Nota. N.A = no aplica puesto que no se especificó o no se habló sobre la edad de los participantes.

Respecto a la condición de los participantes en el momento de los tratamientos, se encontró que el $66 \%$ de los artículos analizados (44) refirieron que la condición era ambulatoria, el 19\% de los artículos (13) trabajaron con pacientes internos en diferentes unidades hospitalarias. Los artículos que trabajaron con participantes internos y ambulatorios fueron el $15 \%$. Estos datos se muestran en la tabla 3 .

Tabla 3. Condición de los participantes.

\begin{tabular}{lc}
\hline Condición Participante & No $^{\circ}$ De Artículos \\
\hline Ambulatoria & 44 \\
Interna & 13 \\
Internos y Ambulatorios & 10 \\
N.A & 4 \\
Total estudios & 71 \\
\hline
\end{tabular}

Nota. N.A = no aplica puesto que no se especificó o no se habló sobre la condición de los participantes.

Tabla 4. Tratamiento farmacológico

\begin{tabular}{lcc}
\hline $\begin{array}{l}\mathrm{N}^{\circ} . \text { de estudios que trabajaron la } \\
\text { adherencia al tratamiento y/o enfermedad }\end{array}$ & 25 & $35 \%$ \\
\hline $\begin{array}{l}\mathrm{N}^{\mathrm{o}} \text {. de estudios que trabajaron } \\
\text { otras temáticas. }\end{array}$ & 46 & $65 \%$ \\
\hline \begin{tabular}{l} 
Total de estudios \\
\hline
\end{tabular} & 71 & $100 \%$ \\
\hline
\end{tabular}

En los artículos analizados se encontró que el $65 \%$ de los estudios refirieron la presencia de medicación en los participantes mientras que en el $32 \%$ de los artículos no se especificó si había medicación o no. Lo anterior permite establecer que la mayoría consideran los medicamentos como agentes necesarios en el momento de iniciar un tratamiento psicoterapéutico.

\section{Adherencia al tratamiento}

Respeto al trabajo que implantaron los artículos analizados con sus referentes terapias, se encontró que el $35 \%$ de ellos (25) describieron haber tendido entre sus ejes principales la importancia de generar adherencia en los pacientes hacia la enfermedad y sus tratamientos (médicos y psicoterapéuticos). El otro $65 \%$ de los artículos se enfocaron en diferentes tipos de temáticas durante su intervención, tales como la regulación emocional, prevención de recaída hospitalaria, creencias, métodos de exposición, entre otras.

\section{Evaluaciones pre-post en los estudios}

Respeto a las intervenciones que realizaron los artículos analizados, se encontró que el $40 \%$ de ellos reportaron haber introducido pre evaluaciones en el tratamiento. El 37\% de estos implementaron evaluaciones pos tratamiento y $18 \%$ incluyeron un seguimiento después de que finalizará la intervención. Por último, se dedujo que el $4 \%$ restante de los estudios no especificaron el contenido de su intervención.

\section{Discusión}

Esta investigación se centró en analizar estudios publicados desde el año 2000 hasta la actualidad respecto a tratamientos psicológicos para la esquizofrenia. La terapia cognitivo comportamental mostró una ventaja significativa respecto a otras terapias: el análisis completo de la funcionalidad de los pacientes y la prevención de recaída hospitalaria son aspectos que resaltan en su desarrollo. La investigación mostró que la terapia cognitivo comportamental, a su vez, trabaja en entrenamientos que son pertinentes al realizar un reajuste social en los pacientes.

En cuanto aspectos contextuales en la historia de aprendizaje, Mortensen y colaboradores (1999) hablaron de antecedentes de la enfermedad en familiares, se encontró que historias de maltrato psicológico en la vida del sujeto pueden llegar a ser un antecedente a la aparición de la enfermedad (Lysaker, Buck y LaRocco, 2007).

Así bien, frente a la producción de artículos científicos que trabajen con trastornos psiquiátricos como la esquizofrenia, en la investigación resaltaron especialmente dos continentes: Europa y América. Esto llevó a cuestionarse 
por el bajo nivel de publicación en los otros continentes; el estudio realizado por Kurihara, Kato, Reverger y Gusti (2006), demuestra la presencia de diferentes creencias místicas frente a la causa de la enfermedad, (voluntad de Dios, brujería, creencias místicas, etc.) que se relacionan con la abstención de toma de tratamiento por parte de pacientes esquizofrénicos.

Varios de los estudios realizados en los últimos años han demostrado la importancia que tiene integrar a las familias desde el inicio del diagnóstico, suministrándoles información suficiente e instruyéndolos en pautas específicas que les ayuden a sensibilizarse frente a lo que está viviendo su pariente, lo cual se percibió en varios de los artículos analizados. Se mostró que la adherencia al tratamiento y/o enfermedad es un factor significativo en el momento de intervenir. Dentro de los artículos seleccionados para el presente trabajo se encontró que más de 1 cuarto (25\%) de ellos consideran que a mayor adherencia se perciba en los pacientes mayor facilidad habrá en el proceso de terapia y por consiguiente mejores resultados. Teniendo en cuenta el análisis que se derivó frente al tipo de terapias identificadas en los artículos analizados, resulta pertinente resaltar que dentro de ese cuarto de muestra que trabajó la adherencia al tratamiento, se encuentra la terapia de tipo cognitivo comportamental, siendo esta la más utilizada en las investigaciones (42\%).

En relación con lo anterior, se encontró que más de la mitad de los artículos analizados mostraron haber tenido como criterio de exclusión que sus participantes se encontraran medicados, especialmente con antipsicóticos y/o antidepresivos, siendo este el $67 \%$ de la muestra, lo cual indica la importancia que tiene el manejo farmacológico en una intervención psicoterapéutica en esquizofrenia.

Después de analizar los distintos artículos incluidos en el presente trabajo, se pudo concluir que a pesar de que la esquizofrenia es una enfermedad que ha sido estudiada desde hace varios años, hay aún muy pocas investigaciones que proporcionen la información suficiente de la misma. Lo anterior permite verificar el estancamiento que existe en las investigaciones de este tipo, lo cual aterroriza e indica la necesidad existente en la actualidad de retomar este tipo de enfermedades y estudiarlas a mayor profundidad.

Respecto a la prevención de recaídas hospitalarias, uno de los estudios analizados escrito por Schmidt, Kraepelin, Janssen y Gaebel, (2009) trabajó en la implementación de un software que evaluaba síntomas del participante y dependiendo los resultados arrojados brindaba recomendaciones respecto ayuda psicosocial y psicoterapéutica, esto con el fin de prevenir recaídas hospitalarias.
Finalmente, la investigación evidencia que el enfoque cognitivo comportamental es aquel que produce mayor cantidad de publicaciones respecto al tratamiento psicoterapéutico para pacientes con esquizofrenia; más allá de esto, también se rescata de este enfoque el tratamiento global del paciente, un adecuado manejo interdisciplinar y un seguimiento continuo del proceso aún años después de haberse terminado.

\section{Referencias}

Addington, J., y Gleeson, J. (2005). Implementing cognitive - behavioural therapy for first-episode-psychosis. The British Journal of psychiatry, 187, 72-76. doi: 10.1192/bjp.187.48.s72

Bauml, J., Frobose, T., Kraemer, S., Rentrop, M., y Pitschel-Walz, G. (2006). Psychoeducation: A Basic Psychotherapeutic Intervention for Patients With Schizophrenia and Their Families. Schizophrenia Bulletin, 32, 1-9. doi: 10.1093/schbul/sb1017

Bechdolf, A., Phillips, L., Francey, S., Leicester, S., Morrison, A., Veith,V. Klosterkötter, J., y McGorry, P. (2006). Recent approaches to psychological interventions for people at risk of psychosis. European Archives of Psychiatry and Clinical Neuroscience Journal, 256, 159-173. doi: 10.1007/s00406-006-0623-0

Belanger, C. (2012). A single- case experiment for an innovative cognitive behavioral treatment of auditory hallucinations and delusions in Schizophrenia. International Journal of psychological studies, 4, 114-122. doi: 10.5539/ijps.v4n1p114

Brenner, H., y Pfammatter, M. (2000). Psychological therapy in schizophrenia: what is the evidence?. Psychiatric Scan, 102, 74-77.doi: 10.1080/15332980902791086

Buckley, L., Pettit, T., y Adams, C. (2004). Supportive Therapy for Schizophrenia. Schizophrenia. Schizophrenia Bulletin. doi: 10.1002/14651858. CD004716.pub3

Cathera, C., Pennb, D., y Ottoa, M. (2005). A pilot study of functional Cognitive Behavioral Therapy (fCBT) for schizophrenia. Schizophrenia RESEARCH, 74, 201-209. doi:10.1016/j.schres.2004.05.002

Christodoulides, T., Dudley, R., Brown, S., Turkington, D., y Beck y colaboradores. (2008). Cognitive behaviour therapy in patients with schizophrenia who are not prescribed antipsychotic medication: A case series. Psychology and Psychotherapy: Theory, Research and Practice, 81, 199-207. doi: 10.1348/147608308X27829

Crawford, M., Killaspy, H., Kalaitzaki, E., Barrett, B., Byford, S., Patterson, S., Soteriou, T., O’Neill, F., Clayton, K., Maratos, A., Barnes, T., Osborn, D., Johnson, T., King, M., Tyrer, P., y Waller, D. (2010). The MATISSE study: a randomised trial of group art therapy for people with schizophrenia. BMC Psychiatry, 10. doi:10.1186/1471-244X-10-65

Cuevas, C. (2006). Terapia cognitivo conductual para los delirios y alucinaciones resistentes a la medicación en pacientes psicóticos ambulatorios. Apuntes de psicología. 24, 267-292.

Dalman, C., Allebeck, P., Cullberg, J., Grunewald, C., y Koster, M. (1999). Obstetric complications and the risk of schizophrenia: a longitudinal study of a national birth cohort. Arch Gen Psychiatry, 56, 234-40. doi:10.1001/archpsyc.56.3.234.

Deane, F., Mercer, J., Talyarkhan, A., Lambert, G., y Pickard, J. (2012). Group Cohesion and Homework Adherence in Multi-Family Group Therapy for Schixophrenia. Australian y New Zealand Journal of Family Therapy, 33, 128-141. doi: http://dx.doi.org/10.1017/aft.2012.15

Díaz, E., Avezuela, R., González M.., Fraile C., y García-Cabeza, I. (2010). Psicoterapia de la Psicosis: De la Persona en Riesgo al Paciente Crónico. (Spanish). Clínica y Salud, 3, 285-297. 
Eack, S. (2012). Integrating Cognitive Behavioral-Based Therapy with an Intersubjective Approach: Addressing Metacognitive Deficits among People With Schizophrenia. Journal of Psychotherapy Integration, 22, 356-374. doi: 10.1037/a0029576

Everitt, B., Steel, C., Tarrier, N., y Wykes, T. (2007). Cognitive Behavior Therapy for Schizophrenia: Effect Sizes, Clinical Models, and Methodological Rigor. Schizophrenia Bulletin, 34, 523-537. doi: 10.1093/ schbul/sbm 114

Fadden, G. (2006). Training and disseminating family interventions for schizophrenia: developing family intervention skills with multidisciplinary groups. Journal of Family Therapy. 28, 23-38. Doi: 10.1111/j.1467-6427.2006.00335.x

Fowler, D. (2011). Cognitive Behavior Therapy for Psychosis: From Understanding to Treatment. Psychiatric Rehabilitation Skills, 4, 199-215. doi: 10.1080/15487760490476183

Fritze, J., Förthner, B., Schmitt, B., y Thaler, U. (2007). Cognitive training adjunctive to pharmacotherapy in schizophrenia and depression: a pilot study on the lateralization hypothesis of schizophrenia and depression and on cognitive therapy as adjunctive to pharmacotherapy. Journal of Cognitive Psychotherapy: An International Quarterl, 19, 45-50. doi: 3185895.

Fusar-Poli, P., y Politi, P. (2008). Paul Eugen Bleuler and the birth of schizophrenia (1908). Am J Psychiatry, 165, 1407. doi: 10.1176/appi. ajp.2008.08050714.

García, I. (2008). Evolución de la psicoterapia en la esquizofrenia. Revista de la Asociación Española de Neuropsiquiatría, 28, 9-25. ISSN: 0211-5735.

García-Martínez, A. T., Guerrero-Bote, V., y Moya-Anegón, F. (2012). World Scientific. Production in Psychology. Universitas Psychologica, 11, 699-717. ISSN: 1657-9267.

García, S., Ruiz, J., Roder, V., y Soler, M. (2007). Social perception training in schizophrenia: A pilot study. International Journal of Psychology and Psychological Therapy, 7, 1-12.

García-Bóveda, R. J. (2007). Habilidades sociales y apoyo social en esquizofrenia. Revista Internacional De Psicología y Terapia Psicológica, 7, 61-71. Recuperado desde http://search.proquest.com/docview/7484 01264 ? accountid $=13250$.

Garety, P., Fowler, D., Freeman, D., Bebbington, P., Dunn, G., y Kuipers, E. (2009). Cognitive-behavioural therapy and family intervention for relapse prevention and symptom reduction in psychosis: randomised controlled trial. Journal of Family Therapy, 192, 412-423. doi: 10.1192/ bjp.bp.107.043570.

Gejman, P. V., y Sanders, A. R. (2012). La etiología de la esquizofrenia. Medicina (Buenos Aires), 72, 227-234

Glyn, S., Marder, S., Liberman, R., Blair, K., Wirshing, W., Wirshing, D., Ross, D., y Mintz, J. (2002). Supplementing clinic-based skills training with manual based communiti suppot sessions: efects on social adjustment of patients with schizophrenia. The American Journal of Psychiatry, 159, 829-837.

Glynnt, S., Cohen, A., y Niv, N. (2007). New challenges in family interventions for schizophrenia. Expert Rev. Neurotherapeutics, 7, 33-43. doi: 11986138

Granholm, E., McQuaid, J., Fauzia, M., Auslander, L., y colaboradores. (2005). A Randomized, Controlled Trial of Cognitive Behavioral Social Skills Training for Middle-Aged and older Outpatients With Chronic Schizophrenia. The American Journal of Psychiatry, 162, 520-529.

Gumley, A., O'grady, M., Mcnay, L., Reilly, J., Power, K., y Norrie, J. (2003). Early intervention for relapse in schizophrenia: Results of a 12-month randomized controlled trial of cognitive behavioural therapy. Psychological Medicine, 33, 419-31. Recuperado desde http://search. proquest.com/docview/204503484 ?accountid=13250.

Guseva, V., Iovlev, B., y Shchelkove, O. (2002). Combined (Individual and Group) Psychodynamic Psychotherapy in the Rehabilitation of Schizophrenic Patients. International Journal of Mental Health, 31, 61- 67.

Hasson-Ohayon, I. (2012). Integrating Cognitive Behavioral-Based Therapy with an Intersubjective Approach: Addressing Metacognitive Deficits among People with Schizophrenia. Journal of Psychotherapy Integration, 22, 356-374. doi: 10.1037/a0029576
Jones, C., Cormac, I., Silveira da Mota Neto, J. I., y Campbell, C. (2010). Cognitive behaviour therapy for schizophrenia (Review). Wiley publishers. doi: $10.1037 / \mathrm{a} 0019115$

Kingdon, D., Rathod, S., Hansen, L., Naeem, F., y Wright, J. (2007). Combining Cognitive Therapy and Pharmacotherapy for Schizophrenia. Journal of cognitive psychotherapy, 21, 28-36. doi: 10.1891/088983907780493278

Klingberg, S., Wölwer, W., Engel, C., Wittorf, A., Herrlich, J., Meisner, C., Buchkremer, G., y Wiedemann, G. (2011). Negative Symptoms of Schizophrenia as Primary Target of Cognitive Behavioral Therapy: Results of the Randomized Clinical TONES Study. Schizophrenia Bulletin, 37, 98-110. doi: 10.1093/schbul/sbr073

Kurihara, T., Kato, M., Reverger, R., y Gusti, R. T. (2006). Beliefs about causes of schizophrenia among family members: A community-based survey in bali. Psychiatric Services, 57, 1795-9. Recuperado desde http://search.proquest.com/docview/213118138?accountid $=13250$

Kurtz, M., y Mueser, K. (2008). A Meta-Analysis of Controlled Research on Social Skills Training for Schizophrenia. Journal of Consulting and Clinical Psychology, 76, 491-504. doi: 10.1037/0022-006X.76.3.491.

La Rotta, E. (2012). Técnicas de intervención dinámica y arte en pacientes con psicopatología severa. Revista de Psicología, 30, 129-168.

Liberman, R. P., Glynn, S., Blair, K. E., Ross, D., y Marder, S. R. (2002). In vivo amplified skills training: Promoting generalization of independent living skills for clients with schizophrenia. Psychiatry, 65, 137-55. Recuperado desde http://search.proquest.com/docview/2206 63145 ? accountid $=13250$.

Lincoln, T., Ziegler, M., Mehl, S., Kesting, M., Llmann, E., Westermann, S., y Rief, W. (2012). Moving From Efficacy to Effectiveness in Cognitive Behavioral Therapy for Psychosis: A Randomized Clinical Practice Trial. Journal of Consulting and Clinical Psychology, 80, 674-686. doi: $10.1037 / \mathrm{a} 002866$

López, P. (1996). Introducción a la bibliometría, PromoLibro, 1, 7-95.

Lysaker, P. H., Davis, L. W., y Beattie, N. (2006). Effects of cognitive behavioral therapy and vocational rehabilitation on metacognition and coping in schizophrenia. Journal of Contemporary Psychotherapy, 36, 25-30. doi:http://dx.doi.org/10.1007/s10879-005-9003-8

Lysaker, P., Buck, K., Rocco, V. (2007). Clinical and psychosocial significance of trauma history in the treatment of schizophrenia. Journal of Psychosocial Nursing y Mental Health Services, 45, 44-51. doi: 17848043

Lysaker, P., y Buck, K. (2006). Narrative enrichment in the psychotherapy for persons with schizophrenia: a single case study. Issues in Mental Health Nursing, 27, 233-247.

Lysaker, P., y Daroyanni, P. (2006). Facilitating the emergence ofvinterpersonal relatedness in I ndividual psychotherapy of schizophrenia: A case study. Bulletin of the Menninger Clinic, 70. doi: 16545032

Lysaker, P., y Hermans, H. (2007). The Dialogical Self in Psychotherapy for Persons With Schizophrenia: A Case Study. Journal of Clinical Psychology, 63, 129-139.

Mak, L., Li, F., y Lee, P. (2007). A Pilot Study on Psychological Interventions with Chinese Young Adults with Schizophrenia. Hong Kong $J$ Psychiatry, 17, 17-23. doi: 10.1002/jclp.20336

McCayand, E., Heather, B., Leszcz, M., Goering, P., Seeman, M., Ryan, K., Johnston, N.,y Vishnevky, T. (2006). A Group Intervention to Promote Healthy Self-Conceptsand Guide Recoveryin First Episode Schizophrenia: A Pilot Study. Psychiatric Rehabilitation Journal, 30 , 105-111. doi: 10.2975/30.2006.105.111

Mohiuddin Ahmed, M., y Boisvert, C. (2003). Multimodal Integrative Cognitive Stimulating Group Therapy: Moving Beyond the Reduction of Psychopathology in Schizophrenia. Professional Psychology: Research and Practice, 34, 644-651. doi: 10.1037/0735-7028.34.6.644

Molloy, C., Conroy, R., Cotter, D., y Cannon, M. (2011). Is traumatic brain injury a risk factor for schizophrenia? A meta-analysis of casecontrolled population-based studies. Schizophr Bull, 37, 1104-10. doi: $10.1093 / \mathrm{schbul} / \mathrm{sbr} 091$

Morrison, A., French, P., Parker, S., Roberts, M., Stevens, H., ... Bentall, R. P.(2007). Threeyear follow-up of a randomized controlled trial of cognitive therapy for the prevention of psychosis in people at ultrahigh risk. Schizophrenia Bulletin, 33, 682-687. doi: 16973786 
Mortensen, P. B., Pedersen, C. B., Westergaard, T., Wohlfahrt, J., Ewald, H., ... Mors, O.(1999). Effects of family history and place and season of birth on the risk of schizophrenia. N Engl J Med, 340, 603-608. doi: 10.1056/NEJM199902253400803

Naeem, F., Kingdon, D., y Turkington, D. (2006). Cognitive behaviour therapy for schizophrenia: Relationship between anxiety symptoms and therapy. Psychology and Psychotherapy, 79, 153-64. Recuperado desde http://search.proquest.com/docview/218895843?accountid=13250. DOI: $10.1348 / 147608305 \times 91538$

Navarrete, J., Fernández, J. A. López, A., Quevedo, R., y Buela, G. (2009). Global psychology: A bibliometric Analysis of Web of Science Publications. Universitas Psychologica, 9, 553-567.

Onwumere, J., Kuipers, E., Gamble, C., Jolley, S., Smith, B., Rollinson, R., Steel, C., Fowler, D., Bebbington, P., Dunn, G., Freeman, D., y Garety, P. (2009). Family interventions in psychosis: a scale to measure therapist adherence. Journal of Family Therapy, 31, 270-283. doi: 10.1111/j.1467-6427.2009.00466.x

Organización mundial de la salud. (1992). CIE-10: Clasificación internacional de enfermedades, décima versión.

Penn, D. L., Roberts, D. L., Combs, D., y Sterne, A. (2007). Best practices: The development of the social cognition and interaction training program for schizophrenia spectrum disorders. Psychiatric Services, 58, 449-51. doi:10.1176/appi.ps.58.4.449

Penn, D. Mueser, K., Tarrier, N., Qloege, A., Cather, C., Serrano,D., y Otto, M. (2004). Supportive Therapy for Schizophrenia: Possible Mechanisms and Implications for Adjunctive Psychosocial Treatments. Schizophrenia Bulletin, 30, 101-112. doi: 15176765.

Pérez-Álvarez, M. (2012). Esquizofrenia y cultura moderna: razones de la locura. (Spanish). Psicothema, 24, 1-9.

Pilling, S., Bebbigton, P., Kuipers, E., Garety, P., Geddes, J., Orbach, G., y Mogan, C. (2002). Psychological treatments in schizophrenia : I. Metaanalysis of family intervention and cognitive behaviour therapy. Psychological Medicine, 32, 763-782. doi: 10.1017/S0033291702005895.

Priebe, S., Savill, M., Reininghaus, U., Wykes, T., Bentall, R., Lauber, C., McCrone, P., Röhricht, F., y Eldridge, S. (2013) Effectiveness and cost-effectiveness of body psychotherapy in the treatment of negative symptoms of schizophrenia - a multi-centre randomised controlled trial. BMC Psychiatry, 13. doi:10.1186/1471-244X-13-26

Ran, M. S., Xiang, M. Z., Chan, C.L., Leff, J., Simpson, P., Huang, M. S., Shan, Y. H., y Li, S.G. (2003). Effectiveness of psychoeducational intervention for rural chinese families experiencing schizophrenia. Social Psychiatry and Psychiatric Epidemiology, 38, 69-75. doi:http:// dx.doi.org/10.1007/s00127-003-0601-z

Read, J., Van Os, J., Morrison, A.P., y Ross, C. (2005). Childhood trauma, psychosis and schizophrenia: A literature review with theoretical and clinical implications. Ada Psychiatrica Scandinavica, 112, 330-350.

Reyes, P. (2007). El potencial relacional del arteterapia en la intervención psicoterapéutica temprana de la psicosis/The art-therapy relational potential in the early psychotherapeutic intervention of psychosis. Arteterapia, 2, 109-118. Recuperado desde http://search.proquest.com/ docview/208960323?accountid=13250.

Roder, V., Mueller, D., y Schmidt, S. (2008). Effectiveness of Integrated Psychological Therapy (IPT) for Schizophrenia Patients: A Research Update. Schizophrenia Bulletin. 37, 71-79. doi: 10.1093/schbul/sb1021

Rogan, A. (2000). "Moving along" in psychotherapy with schizophrenia patients. The Journal of Psychotherapy Practice and Research, 9, 157157. doi: PMC 3330594

Röhricht, F., Papadopoulos, N., Suzuki, I., y Priebe, S. (2009). Egopathology, body experience, and body psychotherapy in chronic schizophrenia. Psychology and Psychotherapy: Theory, Research and Practice, 83, 19-30.doi:10.1348/147608308X342932

Salamero, C., y Manzano, J. (2005). Tratamiento de rituales compulsivos en un caso de esquizofrenia residual: eficacia de la hipnosis. Clínica y Salud, 16, 173-186. doi: 180616104004.
Schinka, J., y Velicer, W. (2003). Handbook of psychology. Research methods in psychology, 2, 3-424.

Schmidt-Kraepelin, C., Janssen, B., y Gaebel, W. (2009). Prevention of rehospitalization in schizophrenia: Results of an integrated care project in germany. European Archives of Psychiatry and Clinical Neuroscience, 259, 205-12. doi: http://dx.doi.org/10.1007/s00406-009-0056-7

Smerud, P., y Rosenfarb, I. (2011). The Therapeutic Alliance and Family Psychoeducation in the Treatment of Schizophrenia: An Exploratory Prospective Change Process Study. Couple and Family Psychology: Research and Practice, 1, 85-91. doi: 10.1037/2160-4096.1.S.85

Spaulding, W., Reed, D., Sullivan, M., Richardson, C., y Weile, M. (2000). Effects of Cognitive Treatment in Psychiatric Rehabilitation. Schizophrenia Bulletin, 25, 657-676. doi: 68588-0308.

Temple, S. (2004). Cognitive therapy for auditory hallucinations in schizophrenia. Journal of Cognitive Psychotherapy, 18, 223-236. doi: http:// dx.doi.org/10.1891/jcop.18.3.223.65647

Twamley, E., Jeste, D., y Bellack, A. (2003). A Review of Cognitive Training in Schizophrenia. Schizophrenia Bulletin. 29, 359-382.

Valencia, M. (2000). Programa de intervención psicosocial para pacientes esquizofrénicos crónicos. Salud mental. 22, 128-137.

Valencia, M., Rascon, M. L., Juarez, F., Escamilla, R., Saracco, R., y Liberman, R. P. (2010). Application in mexico of psychosocial rehabilitation with schizophrenia patients. Psychiatry, 73, 248-63.doi: http:// dx.doi.org/10.1521/psyc.2010.73.3.248

Van der Qaag, M., Kern, R., Van den Bosch, R., y Liberman, R. (2002). A Controlled Trial of Cognitive Remediation in Schizophrenia. Schizophrenia Bulletin. 28, 167-176.

Vaskinn, A., Sundet, K., Friis, S., Simonsen, C., Birkenaes, A., Jonsot, H., Ringen, P., y Andreassen, O. (2008). Emotion perception and learning potential: Mediators between neurocognition and social problem-solving in schizophrenia? Journal of the International Neuropsychological Society, 14, 279-288. doi: http://dx.doi.org/10.1017/ S1355617708080314

Vestergaard, M., Pedersen, B., Christensen, J., Madsen, K., Olsen J., y Mortensen, P. (2005). Febrile seizures and risk of schizophrenia. Schizophr Res, 73, 343-9. doi: 10.1016/j.schres.2004.07.004

Virgil, L., y Gregory, J. (2010). Cognitive-behavioral Therapy for schizophrenia: applications to social work practice. Social work in mental health, 8, 140-159. doi: http://dx.doi.org/10.1080/15332980902791086

Walkup, J., Wei, W., Sambamoorthi, U., Crystal, S., y Yanos, P. (2006). Provision of Psychotherapy for a Statewide Population of Medicaid Beneficiaries With Schizophrenia. Psychological Services, 3, 227-238. doi: 10.1037/1541-1559.3.4.227

Weisman, A. (2005). Integrating culturally based approaches with existing interventions for hispanic/latino familias doping with schizophrenia. Educational Publishing Foundation, 2, 178-197.

Weisman, A., Duarte, E., Koneru, V., y Wasserman, S. (2006). The development of a culturally informed, family focused treatment for schizophrenia. Family Process, 45, 171. doi: 10.1111/j.15455300.2006.00089.x

Wolwer, W., y Frommann, N. (2011). Social-Cognitive Remediation in Schizophrenia: Generalization of Effects of the Training of Affect Recognition (TAR). Schizophrenia Bulletin, 37, 63-70. doi:10.1093/ schbul/sbr071.

Zimmermann, T., Favrod, V.H., y Trieu, V. (2005). The effect of cognitive behavioral treatment on the positive symptoms of schizophrenia spectrum disorders: A meta-analysis. EL SEVIER. 77. doi: org/10.1016/j. schres.2007.12.468

Zygmunt, A., Olfson, M.., Boyer, C., y Mechanic, D. (2002). Interventions to improve medication adherence in schizofrenia. The American Journal of psychiatry, 159, 1653-1664. doi: 0.1176/appi.ajp.159.10.1653 
